\title{
Statistical Topography of Glassy Interfaces
}

\author{
Chen Zeng \\ Department of Physics, Rutgers University, Piscataway, NJ 08855, USA \\ Jané Kondev \\ Department of Physics, Brown University, Providence, RI 02912, USA \\ D. McNamara and A. A. Middleton \\ Department of Physics, Syracuse University, Syracuse, NY 13210, USA
}

(October 17, 2018)

\begin{abstract}
Statistical topography of two-dimensional interfaces in the presence of quenched disorder is studied utilizing combinatorial optimization algorithms. Finite-size scaling is used to measure geometrical exponents associated with contour loops and fully packed loops. We find that contour-loop exponents depend on the type of disorder (periodic "vs" non-periodic) and they satisfy scaling relations characteristic of self-affine rough surfaces. Fully packed loops on the other hand are unaffected by disorder with geometrical exponents that take on their pure values.
\end{abstract}

Elastic manifolds in random media are used to model various condensed-matter systems with quenched disorder, including flux-line arrays in dirty type-II superconductors [1], and charge density waves [2]. These disparate systems exhibit a common feature, namely a low temperature glassy phase in which static and dynamic properties are dominated by the disorder. One of the simplest models used to study this glassy phase is given by a 2-dimensional isotropic interfaces embedded in a 3 -dimensional disordered medium. The interface Hamiltonian in the continuum limit is

$$
H(\{h(\mathbf{x})\})=\int d^{2} \mathbf{x}\left[\frac{K}{2}|\nabla h(\mathbf{x})|^{2}+V[\mathbf{x}, h(\mathbf{x})]\right],
$$

where $h(\mathbf{x})$ is the height of the interface above a flat basal plane indexed by a 2 -dimensional vector $\mathbf{x}$, and $K$ is the elastic stiffness constant. Depending on whether the otherwise uncorrelated random potential $V[\mathbf{x}, h(\mathbf{x})]$ is non-periodic or periodic along the height direction, the interface is usually referred to as a random manifold or a random elastic medium, respectively [3].

Much of the analytical understanding of these glassy interfaces comes from functional renormalization group calculations [4] [ The random manifold is found to have a zero-temperature fixed point characterized by a roughness exponent $\zeta \approx 0.41$ 昍, i.e., the width $(W)$ of the interface, as measured by the square root of the height variance, scales with its lateral linear size $(L)$ as $W \sim L^{\zeta}$. The random elastic medium on the other hand exhibits a low-temperature fixed line below a glass transition. Along this line the interface is super-rough $(W \sim \ln (L))$, while in the high temperature phase it is marginally rough $(W \sim \sqrt{\ln (L)})$ [5]. Recent numerical studies of the exact ground-state roughness based on combinatorial optimization algorithms strongly support the above picture [6].
In this letter, we apply further refined implementations of these polynomial-in-time algorithms to study the topography [7] of disordered interfaces at zero temperature. In contrast to the usual paradigm of a rugged landscape [8] where the surface morphology is described in terms of height-height correlations, we instead focus on extended, non-local features of the random geometry as expressed by contour loops (i.e., lines of constant height) and fullypacked loops (defined below). We measure different geometrical exponents for the two types of loops and check various scaling relations among them (see Eq. (5)). This approach has proven to be very useful in characterizing the morphology of rough surfaces whenever the complete height profile is available [9].

From the results obtained for contour loops our main conclusion is that the glassy interfaces studied are selfaffine and rough with roughness exponents in good agreement with theoretical findings. The study of fully packed loops on the other hand addresses the question of the effect of quenched disorder on critical fluctuations. Namely, the discrete interface models we employ map to disordered fully packed loop models. In the absence of disorder this loop model is critical and its exponents have been calculated exactly using Bethe ansatz and Coulomb gas techniques [10]. Unexpectedly, here we find that the values of the pure exponents are unaffected by disorder.

a. Models and algorithms To simulate both random and random-periodic interfaces, we consider a simple cubic lattice with random bond weights (energies) and its directed (111)-interface. This interface, to be precise, is defined on the dual lattice with each elementary plaquette (a square) intersecting a single bond of the simple cubic lattice. The cost of such an interface is defined to be the sum of the weights of all the bonds that it cuts. In the case of the random manifold the integervalued weight of each bond is chosen independently and 
uniformly in the interval $[0,1000]$. When simulating a random elastic medium the random weights are sampled in the same way with the important difference that the weights of all the bonds directly above one another along the (111) direction are set equal. In other words, the bond disorder is uncorrelated for the former and periodic along the height direction for the latter, reflecting the intrinsic periodicity of the random elastic medium 3.

The problem of finding the ground state of an interface in the presence of quenched disorder is that of minimizing its total energy cost. Employing max-flow-min-cut and minimum-cost perfect-matching algorithms, for the case of random disorder and random-periodic disorder respectively, enables us to determine the exact ground state interface in time that grows only polynomialy with $L$. This allows us to simulate relatively large system sizes for many disorder realizations.

The max-flow-min-cut algorithm interprets the simple cubic lattice as a directed flow network. Each bond of the lattice is directed along the (111) direction, and its random weight specifies the maximum amount of flow that can be accommodated in this bond. The algorithm finds the interface of minimum cost by searching instead for the maximum flow (with flow conservation) that can be sustained between the bottom and top layers with the interface sandwiched in between; see Fig.1. The algorithm works because the interface of minimum cost is the bottleneck through which all flow must pass.

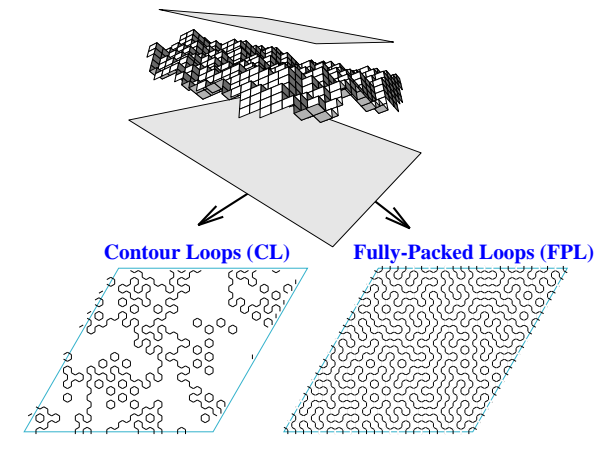

FIG. 1. (111) interface of a simple cubic lattice and its two loop representations. An example of a ground-state interface confined between two flat (111) layers is shown. The level set at mean height consists of closed contour loops due to periodic boundary conditions. Also shown is the fully-packed-loop (FPL) representation of the same interface (see text for details of construction).

Next we note that the (111) projection of the interface gives a rhombus tiling of the plane, which is also equivalent to a complete (perfect) dimer covering of a hexagonal lattice. The hexagonal lattice $(\mathcal{L})$ in question is the dual of the triangular lattice formed by the vertices of the rhombi, while the dimers lie perpendicular to their short diagonals 11. This mapping is purely geometrical in nature, but when the disorder is periodic it facilitates the task of finding the interface of minimum cost. Namely, the periodic disorder can be represented entirely by random bonds on $\mathcal{L}$, and the minimization problem becomes one of finding the perfect matching (dimer covering) with minimum cost. Detailed descriptions of both algorithms can be found in Ref. 12].

b. Geometric exponents and scaling Given the exact shape of the (111)-interface its topography is completely characterized by a contour plot with the level spacing equal to a single step of the the discrete height. The contour plot consists of contour loops which live along the bonds of the hexagonal lattice $\mathcal{L}$. The contours are closed due to periodic boundary conditions which we impose in both lateral directions. For example, in Fig.1 we have drawn all the contour loops at mean height, for the (111) interface shown. The union of all the contour loops for different realizations of disorder is the contour loop ensemble.

Apart from this natural contour-loop characterization, there exists yet another interesting loop representation of the (111)-interface, the fully-packed loops (FPL). These loops owe their existence to the one-to-one mapping between a (111)-interface and a complete dimer covering of the hexagonal lattice $\mathcal{L}$. By removing the bonds of $\mathcal{L}$ that coincide with the dimers, we are left with a configuration of fully-packed loops, as shown in Fig.1, where every site of $\mathcal{L}$ belongs to one and only one loop. A physical realization of FPL are the magnetic domain walls in the ground state of the Ising antiferromagnet on the triangular lattice [1]. FPL models of general loop fugacity in the absence of disorder have been studied recently [10] and were shown to be critical for values of the loop fugacity that does not exceed two. The interface-FPL mapping thus allows us to consider the effect of quenched disorder on the critical FPL model on the honeycomb lattice with fugacity equal to one.

Following Ref. 91, we consider the fractal dimension of a loop $D$, the loop distribution exponent $\tau$, the loop correlation exponent $x_{l}$, and finally the interface roughness exponent $\zeta$. These geometrical exponents are used to characterize the morphology of an interface which is statistically invariant under the rescaling $h(\mathbf{x}) \rightarrow b^{-\zeta} h(b \mathbf{x})$, where $b>1$ is an arbitrary rescaling parameter; such interfaces are termed self-affine.

The natural quantities associated with a loop are its length $s$ and the radius of gyration $R$, both measured here in units of the lattice spacing. For the ensemble of loops we define $n(s, R)$, the number density of loops of length $s$ and radius $R$. Contour loops of a self-affine interface have no characteristic length scale and we anticipate a scaling form for the number density:

$$
n(s, R)=s^{-(1 / D+\tau)} f\left(s / R^{D}\right) .
$$


Integrating $n(s, R)$ over all radii gives the number density of loops of length $s$,

$$
n(s) \sim s^{-\tau}
$$

which we use to extract the exponents $\tau$ and $D$ (see the following section).

Yet another measure that was introduced in Ref. [9] is the loop correlation function $g(r)$ which gives the probability that two points separated by distance $r$ belong to the same loop. Just like in the case of the number density of loops we anticipate a power law

$$
g(r) \sim r^{-2 x_{l}},
$$

where $x_{l}$ is the loop correlation exponent.

In Ref. [9] scaling relations were derived for the loop exponents $\tau, D$, and $x_{l}$ assuming a self-affine interface of roughness $\zeta$ :

$$
\begin{aligned}
D & =2-x_{l}-\zeta / 2 \\
\tau & =1+(2-\zeta) / D .
\end{aligned}
$$

These results follow from sum rules for the average loop length and the average number of large loops (i.e., those with radii comparable to $\rho$ ) inside an area of linear size $\rho$. Eq. 同 tells us that there are only two independent exponents so measuring all four and checking the validity of the scaling relations provides an important consistency check on the assumed self-affine nature of the rough interface. Furthermore, the second scaling relation suggests a method for measuring the roughness exponent directly from the loop data. Namely, integrating $n(s, R)$ over all $s$ and making use of $D(\tau-1)=2-\zeta$, we find

$$
n(R) \sim R^{\zeta-3}
$$

for the number density of loops of radius $R$.

c. Numerical results We first describe our results for the random elastic medium. Four different sample sizes, $L=72,120,240$, and 480 were simulated with $10^{4}$ disorder realizations for each size.

The fractal dimension $D$ and loop exponent $\tau$ can be simultaneously extracted from $n(s)$ using a finitesize scaling (FSS) form $n(s)=s^{-\tau} f_{n}\left(s / L^{D}\right)$, which follows from Eq. 3. In order to minimize the statistical noise at large $s$, we consider instead the cumulative number density $N(s) \equiv \int_{\tilde{s}>s} n(\tilde{s}) d \tilde{s}$, with the scaling form $N(s)=s^{1-\tau} f_{N}\left(s / L^{D}\right)$. Results are summarized in Fig 2. The power-law scaling regime is evident over three decades in loop length $(40<s<40000)$. The deviations from scaling at small and large $s$ are attributed to the lattice cutoff and finite lattice size, respectively. The best data collapses yield $D=1.46 \pm 0.01$ and $\tau=2.32 \pm 0.01$ for CL, and $D=1.75 \pm 0.01$ and $\tau=2.15 \pm 0.01$ for FPL.

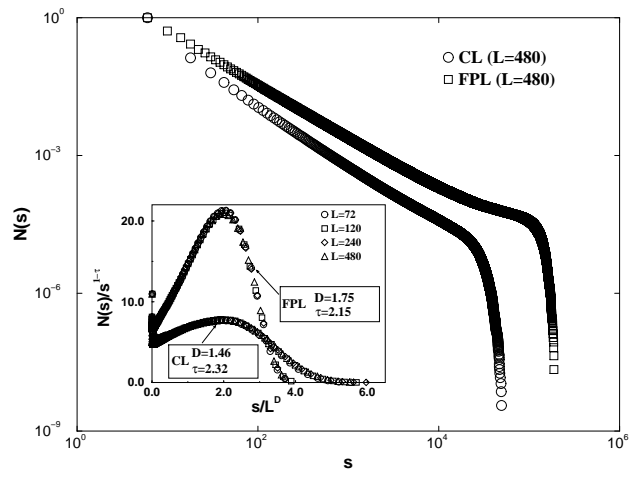

FIG. 2. Cumulative number density $N(s)$ (normalized by total loop number), for CL and FPL; $L=480$. FSS analysis for $L=72,120,240$, and 480 , is shown in the inset and used to determine exponents $D$ and $\tau$ (see text). $N(s)$ is binned in intervals of $0.08 s$ at successive $s$ to estimate the systematic errors which are smaller than the symbols shown.

We have also computed the loop correlation function $g(r)$, from which the exponent $x_{l}$ is extracted using a scaling form $g(r)=r^{-2 x_{l}} f_{g}(r / L)$. The best data collapses, shown in the inset of Fig. 3, yield $x_{l}=0.50 \pm 0.01$ and $x_{l}=0.25 \pm 0.01$ for CL and FPL, respectively. An exact value $\left(x_{l}^{\text {exact }}=1 / 2\right)$ of the loop correlation exponent for CL was proposed for self-affine rough surfaces independent of the roughness [9]. Our results (see Table I) therefore provide further evidence of the universal nature of $x_{l}$ for contour loops.

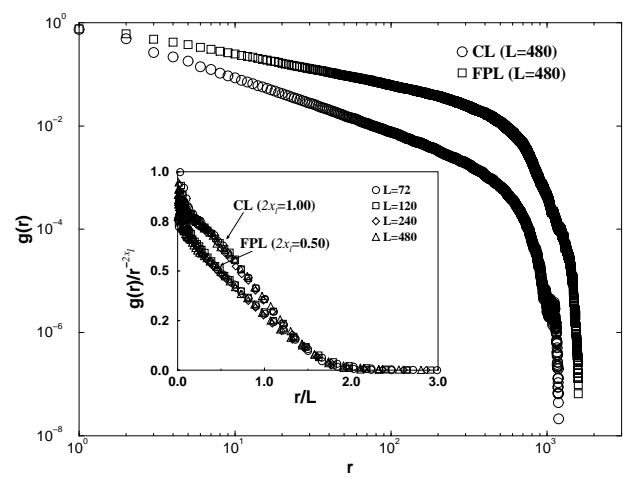

FIG. 3. Loop correlation function $g(r)$ for both CL and FPL; $L=480$. FSS plots are shown in the inset; systematic errors estimated as in Fig. 2 are smaller then the symbols shown.

From Eq. 6 it follows that the roughness exponent $\zeta$ can be obtained from $n(R)$. Once again we consider the cumulative number density $N(R)$ for which we propose the scaling form: $N(R)=R^{\zeta-2} f_{N}(R / L)$. The results of the FSS analysis are given in Fig 1 which yields $\zeta=0.08 \pm 0.01$ and $\zeta=0.00 \pm 0.01$ for CL and FPL, respectively. 


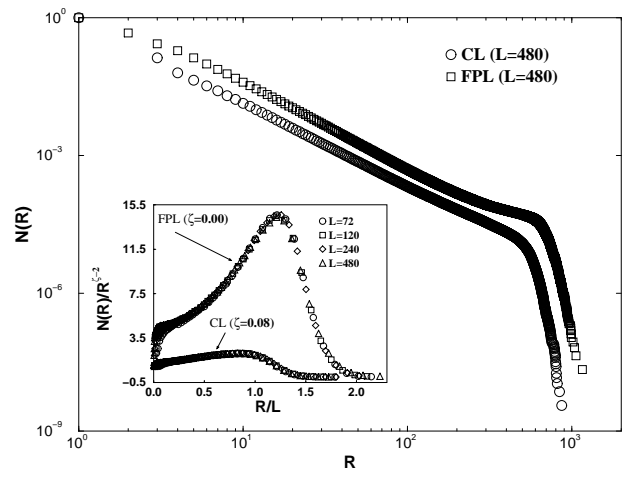

FIG. 4. Cumulative number density $N(R)$ (normalized by total loop number) for both CL and FPL; $L=480$. FSS plots are shown in the inset; systematic errors estimated as in Fig. 2 are smaller then the symbols shown.

Given the numerical values of the geometrical exponents in the case of the random elastic medium, which are summarized in Table I, it is straightforward to check that the scaling relations, given by Eq. 5 , hold for both CL and FPL. Although the small non-zero roughness $\zeta=0.08$ appears to be in disagreement with the super-rough scenario $(\zeta=0)$, it can in fact be understood as an effective exponent due to the extra log-divergence of the interface width. We therefore expect this effective roughness to approach zero with increasing system size as $1 / \ln L$.

We also carried out numerical simulations for the random manifold. Our results for the geometrical exponents are summarized in Table I. They were obtained in the exact same fashion as in the case of the random elastic medium using a FSS analysis for $L=64,72,96$, and 150 , with the interfaces confined between two boundary layers separated by $H=55,58,65$, and 78 , respectively; see Fig. 1. For each system size $6 \times 10^{3}$ disorder realizations were simulated. Our three main conclusions are: First, the roughness exponent $\zeta=0.40(2)$ obtained from $N(R)$ is in excellent agreement with that obtained from a more traditional approach that relies on measuring height fluctuations [6]. Second, as in the case of the random elastic medium, geometric exponents of both CL and FPL satisfy scaling relations given in Eq. (5) giving strong support to the claim that this glassy interface is self-affine. Finally, and quite surprisingly, the geometric exponents for FPL remain unchanged from the randomperiodic case. Moreover, their values agree, within statistical errors, with those obtained in the absence of disorder, where the interface is marginally rough due to entropic fluctuations [10].

In conclusion, we have measured the geometrical exponents of contour loops and fully packed loops associated with the ground states of interfaces in the presence of quenched random and periodically-random disorder. The contour loop results are consistent with the ground state interfaces being self-affine and rough, with roughness ex- ponents in agreement with renormalization group calculations. The geometrical exponents for the fully packed loops were found to be unaffected by the disorder. This discovery is rather unexpected in light of the fact that there is a 1-1 mapping between the two loop types, and it calls for a more detailed study of disordered fully packed loop models with general loop weights.

We thank J. Cardy, C.L. Henley, J. Jacobsen, P. Leath, C. Marchetti, and V. Pasquier for useful discussions. We acknowledge support by the NSF through grants DMR9214943 (CZ), DMR-9357613 (JK), and DMR-9702242 and Alfred P. Sloan Fellowship (AAM).

TABLE I. Geometric exponents of both contour loops (CL) and fully-packed loops (FPL). Rational numbers are the proposed exponents.

\begin{tabular}{|ll|}
\hline \hline Random Elastic Medium & \\
\hline \hline Contour Loops (CL) & Fully-Packed Loops (FPL) \\
\hline$D=1.46 \pm 0.01(3 / 2)$ & $D=1.75 \pm 0.01(7 / 4)$ \\
\hline$\tau=2.32 \pm 0.01(7 / 3)$ & $\tau=2.15 \pm 0.01(15 / 7)$ \\
\hline$x_{l}=0.50 \pm 0.01(1 / 2)$ & $x_{l}=0.25 \pm 0.01(1 / 4)$ \\
\hline$\zeta=0.08 \pm 0.01(0)$ & $\zeta=0.00 \pm 0.01(0)$ \\
\hline \hline Random Manifold & \\
\hline \hline Contour Loops (CL) & Fully-Packed Loops (FPL) \\
\hline$D=1.31 \pm 0.02(?)$ & $D=1.74 \pm 0.01(7 / 4)$ \\
\hline$\tau=2.19 \pm 0.02(?)$ & $\tau=2.15 \pm 0.01(15 / 7)$ \\
\hline$x_{l}=0.49 \pm 0.02(1 / 2)$ & $x_{l}=0.25 \pm 0.01(1 / 4)$ \\
\hline$\zeta=0.40 \pm 0.02(?)$ & $\zeta=0.01 \pm 0.01(0)$ \\
\hline \hline
\end{tabular}

[1] G. Blatter et al, Rev. Mod. Phys. 66, 1125 (1994).

[2] G. Grüner, Rev. Mod. Phys. 60, 1129 (1988).

[3] L. Balents, Lecture Notes (unpublished).

[4] D.S. Fisher, Phys. Rev. Lett. 56, 1964 (1986).

[5] J.L. Cardy and S. Ostlund, Phys. Rev. B 25, 6899 (1982); J. Toner and D. P. DiVincenzo, Phys. Rev. B 41, 632 (1990); Y.-C. Tsai and Y. Shapir, Phys. Rev. Lett. 69, 1773 (1992).

[6] A.A. Middleton, Phys. Rev. E. 52, R3337 (1995); C. Zeng et al, Phys. Rev. Lett. 77, 3204 (1996); H. Rieger and U. Blasum, Phys. Rev. B. 55, R7394 (1997).

[7] For a review of statistical topography see M.B. Isichenko, Rev. Mod. Phys. 64, 961 (1992).

[8] D. Sherrington, to be published in Physica D (1997).

[9] J. Kondev and C.L. Henley, Phys. Rev. Lett. 74, 4580 (1995); J. Kondev, D.G. Salinas, and C.L. Henley, unpublished.

[10] H.W.J. Blöte and B. Nienhuis, Phys. Rev. Lett. 72, 1372 (1994); M.T. Batchelor et al, Phys. Rev. Lett. 73, 2646 (1994); J. Kondev et al, J. Phys. A 29, 6489 (1996).

[11] H W J Blöte, and H J Hilhorst, J. Phys. A: Math. Gen. 15, L631 (1982). B. Nienhuis et al, J. Phys. A: Math. Gen. 17, 3559 (1984); C. Zeng and C.L. Henley, Phys. Rev. B. 55, 14935 (1997).

[12] U. Derigs, Programming in Networks and Graphs, Springer-Verlag, New York (1988); H. Rieger, Lecture Notes in Physics, Springer-Verlag, New York (1997). 